Vol.01/ No. 01

Pages: $14-23$

http://irojournals.com/jscp/

DOI: https://doi.org/10.36548/jscp.2019.1.002

\title{
PERFORMANCE ANALYSIS OF GRANULAR COMPUTING MODEL IN SOFT COMPUTING PARADIGM FOR MONITORING OF FETAL ECHOCARDIOGRAPHY
}

\author{
Dr. A. Sathesh, \\ Professor, Department of Electronics and Communication Engineering, \\ Eritrea Institute of Technology, \\ Eritrea. \\ Email: $\underline{\text { Sathesh4you@gmail.com }}$
}

\begin{abstract}
The monitoring of fetal heart being essential in the second trimester of the prenatal periods. The abnormalities in the child heart rate has to be identified in the early stages, so as to take essential remedies for the babies in the womb, or would enable the physician to be ready for he complication on the delivery and the further treatment after the baby is received. The traditional methodologies being ineffective in detecting the abnormalities leading to fatalities, paves way for the granular computing based fuzzy set, that requires only a limited set of data for training, and helps in the eluding of the unwanted data set that are far beyond the optimal. Further the methods performance is analyzed to evident the improvement in the fetal heart rate detection in terms of prediction accuracy and the detection accuracy.
\end{abstract}

Keywords: Granular computing, Soft computing, Fuzzy sets , Training data set, Fetal heart rate , Prediction accuracy and Detection accuracy

\section{Introduction}

In recent days, efficacious techniques or practices in dealing with the huge amount of information gathered has become an essential necessity [6] and the granular computing has become one of the popular technique that enhances the decision support by procedures of data abstraction and the deriving of the knowledge from the information or data for solving the complex problems [5], the word granule or the information, are the entities that hold the numeric values that are grouped to gather due to the similarity they poses or the proximity or their functionality or the coherency. The granular computing is usually a theoretical perspective, as the granular computing also termed as the information granularity or the information granulation related to the fuzzy set was 
Journal of Soft Computing Paradigm (JSCP) (2019)

Vol.01/ No. 01

Pages: $14-23$

http://irojournals.com/jscp/

DOI: https://doi.org/10.36548/jscp.2019.1.002

initially defined as the subset of the computing words [19] it is the umbrella term that holds the strides, theories, techniques and the tools to solve the problem such are complex, the granule computing shows a predicting accuracy and eluding the solution that are far away from the optimal solutions [12]. This granular computing proceeds with two steps (i) granulation and (ii) organization, where the granulation breaks the information into subparts to discover the knowledge and the organization refers to building it back. The granular computing eluding the unwanted solutions that are far beyond the optimal and arriving to solution that are beneficial in terms of the prediction and detection than the traditional methods, the fuzzy set based granular computing does not require more number of dataset to be trained as the conventional methods that require innumerable datasets to train in order to prove its accuracy,

The paper concentrates on the monitoring of the fetal echo cardiology (FEC), for the identification of the abnormalities in the heart rate of the child in the womb, where the early detection would allow the physician to start with the treatment from the womb or enable him to get ready for the complications on the delivery or with the treatment after the birth.

The fuzzy based analysis based on the granular computing, is utilized in the detection of the heart rate of the child in the fetus, overcoming the traditional way of FEC that are the CTG [20] and the HRV [21].

The remaining paper is organized with the section 2 related works and the granular computing and the fetus heart rate monitoring, section 3 the proposed work section 4 the analysis of prediction using the fuzzy set based on the granular computing and section 5. Conclusion.

\section{Related works}

Skowron et al [1] proposes the granular computing that gathers the concepts from the data generated into a clusters, set of rules aggregation and operation etc. to control, predict and bound the behavior of the system. The author details the proposal that investigates the challenges in the frame work of the granular computing. Bargiela, et al [2] presents the study that details the motivation behind the formation of the information granule and the casting of the granular computing as the as the structured combination of the algorithmic and non-algorithmic that imitates the human intelligence in combination with the knowledge gathered from the information. Song et al [3] present a study with the scope of providing the insights of the granular neural networks, where the granules are the group of information that are similar to be useful in the fields of knowledge prediction and the recognition of patterns. Bargiela et al [4] the information inaccuracies in the traditional method could be overcome using the information 
Journal of Soft Computing Paradigm (JSCP) (2019)

Vol.01/ No. 01

Pages: $14-23$

http://irojournals.com/jscp/

DOI: https://doi.org/10.36548/jscp.2019.1.002

granules that gather the knowledge from the data gathered, to have closer prediction of the data aggregated. Pal, et al [5] the author presents the granulation based information processing model for the soft computing paradigm, for the development of the computational tools that are used in solving the complex problems. Skowron et al [6] the author analysis the benefits of utilizing the granular computing that involves the information granules, to a have a more precise prediction and knowledge discovery from information's gathered. The adaptive judgment along with the granular computing are analyzed for efficient management, risk assessment and the benefit analysis. Livi, et al [7], the paper reviews the unusual aspects related to the non-geometric pattern analysis and discusses the difficulties in the data granulation and the necessity of the granular computing. Costa, et al [8] the paper proposes the advanced techniques in the fetal heart rate analysis and the fatalities that would happen, if not properly diagnosed. Gupta et al [9] the fuzzy based the granular computing in identifying the student's strategies in the examination is proposed in the paper. Guo, et al [10] the paper proposes the granular support vector machine to obtain the satisfactory generalization performance. The granular computing solves the low efficiency learning problem that exists in the traditional methods. Gerardo et al [11] is the study analysing the granular computing and traditional neural network and presents the advancement in term of prediction accuracy in the granular computing. Cruz-Vega et al [12] the proposed method of the granular modeling with the fuzzy aptitude based eluding the unnecessary evaluation of the solutions that are not in proximity with the optimal solutions. Fujita et al [13] the paper presents the granular computing integrated with the system for the resilience analysis to have a rapid decision making on the time of disasters Kozik et al [14] the author utilizes the information granules for the detection of the cyber-attacks and discusses the feasibility of utilizing the granules in the cyber-attacks. Bailey et al [15] the analytical model for processing of the input data stream is investigated to input, output and the action, for the granular performance analysis of metrics and diagnostics in trouble shooting. Cabrerizo et al [16] the author presents the frame work of the granular computing for deriving the computational elements from the natural languages. Anshuman et al [17] the edge of the communication networks are monitored using the multi-granular computing enabled with the learning feedback. Zhu et al [18] the author proposes an approach to the designing and the analysing of the granular input space for the utilizing the granular modeling.

\section{The Necessity of Fetal Echo-Cardiology}

The fetal echocardiography's a test performed in the second trimester of the pregnancy. The test is done for the doctor to have a better visualization of the baby's heart in the womb. The test is performed by analysing the sound waves of the heart in the fetus that is the echo of the heart, the echo observed is analyzed and presented out as the echocardiogram, the graphical images acquired is to describe the working of the heart of the baby. This enables the doctor to know about the rate of the blood flow in the heart and the malfunctions or the abnormalities in the heart.

ISSN: 2582-2640 (online) 
Journal of Soft Computing Paradigm (JSCP) (2019)

Vol.01/ No. 01

Pages: $14-23$

http://irojournals.com/jscp/

DOI: https://doi.org/10.36548/jscp.2019.1.002

The traditional CTG used does not show much difference between the normal and the abnormal heart rate of the fetus, so the paper goes for the granular computing and the fuzzy based model to identify the rate of the heart beat in the fetus that is normal and abnormal. The fig. 1 shows the fetal echo cardiology obtained using the conventional method.

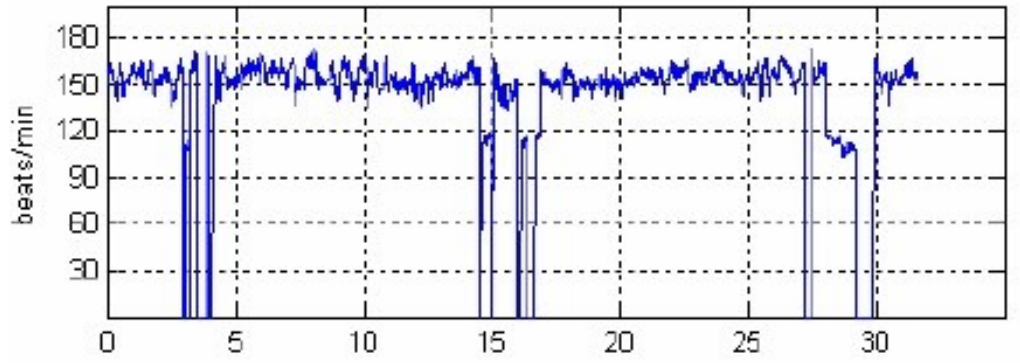

Fig .1 conventional FEC by CTG [21]

The conventional method does not show any differences between the normal rate of the heart and the identification of the abnormalities, so the proposed method helps in the identifying the abnormalities in the heart rate of the fetus.

\section{Granular and the Fuzzy Based Model}

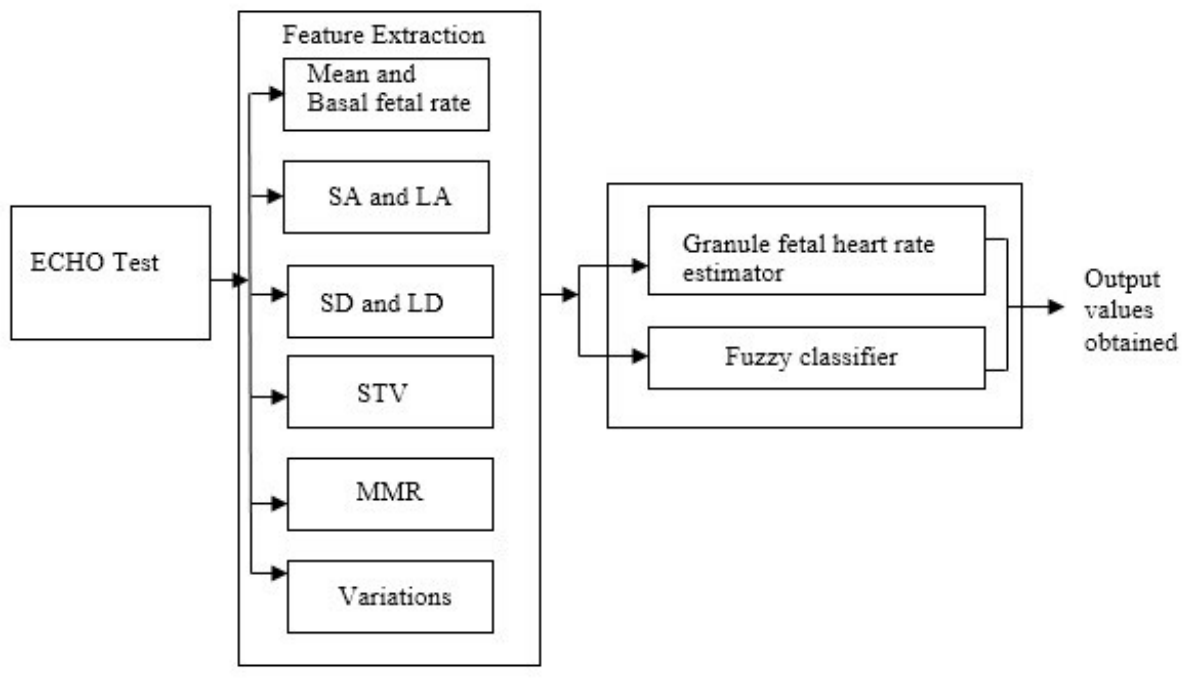

Fig .2 Proposed Granular and Fuzzy based Modeling 
Journal of Soft Computing Paradigm (JSCP) (2019)

Vol.01/ No. 01

Pages: $14-23$

http://irojournals.com/jscp/

DOI: https://doi.org/10.36548/jscp.2019.1.002

The fig 2 gives the granular and the fuzzy logic based module for the detection and the classification of the abnormalities in the fetal-ECHO, the echo from the test results obtained are analyzed to identify the abnormalities in the babies heart beat and the blood flow. The results of the Echo-test are gathered and the features are extracted and the analyzed using the granular and the fuzzy based module for the identification of the abnormalities and then predicts the output. So the proposed process proceeds as three stages as data gathering, feature extraction and analysis that helps in the detection of the unusual heat beat.

(i) Information Collection: The information of the echo test were gathered from 50 women, who were under the observation in the unit of obstetrics and gynecology of a private hospital. The pregnancy was a singleton pregnancy and the gestational age of the pregnancy was observed to be between the 18 to 24 months. The ECHO-test collected were taken as the initial input of the proposed model.

(ii) Feature Extraction: From the data set collected the, based on the echo-test that were performed of 50 women under the observation the following features were extracted using the discrete wavelet transformation [21]. The ECHO-test results acquired are subjected to extract the features that are related to the identification of abnormalities in the heart rate and the blood flow of the babies insides the mother's womb. Some of the features extracted are mean and the basal fetal rate, the short and the long acceleration's, the short and the long decelerations, the short term variations, the mean minute range ,the lowest and the highest variations in the heart beat. The fetal heartbeat observed using the FEC is decomposed to extract its features using the discrete wavelet transform. The discrete wavelet transformation applied decomposes the signals of the fetal heart rate into sub bands using the MATLAB functions. And computed calculating the mean, and the variance. Where mean gives the average value and the variance gives the sum of square of the difference between each values in the distribution from the mean, divided by the number of values in the distribution. The equation (1) and (2) gives the formula applied in estimating the mean and the variance.

$$
\begin{array}{r}
m=\left(\frac{1}{n}\right) \sum_{i=1}^{n} x_{i} \\
v=\frac{1}{n-1} \sum_{i=1}^{n}\left(x_{i}-m\right)^{2}
\end{array}
$$


Journal of Soft Computing Paradigm (JSCP) (2019)

Vol.01/ No. 01

Pages: $14-23$

http://irojournals.com/jscp/

DOI: https://doi.org/10.36548/jscp.2019.1.002

The signals decomposed using the DWT were extracted with the six values for every scale subjected to decomposition, the values of the six features extracted from fetal heart rate were used as the inputs to the module based on the granule and the fuzzy logic.

(iii) Granule and Fuzzy based Classifier: The features extracted were given as input to the granule fetal heart rate estimator, to recognize the differences and the consistency in the information gathered. In order to quantize the values obtained from the features extracted, to gain the valuable information's, the granule fetal heart estimator performs the quantization of the values obtained from the discrete wavelet transformation to gain the necessary meaning full information regarding the heart rate of the baby to identify whether its functioning is normal or abnormal the values are quantized between zero or one based on the threshold fixed on the normal and the abnormality of the fetal heart rate respectively . Further the extorted features values are assigned as the training data set to the fuzzy classifier employs the fuzzy-KNN in identifying or categorizing the observed values between the normal or the abnormal. The steps below explains the fuzzy-KNN in classification of the fetal heart rate between the normal and the abnormal conditions identifying the complications in the infant.

Step1: The values extracted based on the features observed are converted into the training data sets.

Step2: The membership degree for the values obtained for the features are calculated applying the Gaussian membership function,

Step3: Evaluate distance for each samples acquired

Step4: Gather the k samples at the proximity in the training according to the distance calculated.

Step5: Add the membership vectors of the examines acquired and retrieve the un-normalized values Step6: obtain the normalized fuzzy vector.

So the proposed process, involving both the granule estimator for the heart rate and the fuzzy classifier, helps in the identification of the abnormalities of a child before birth, enabling the physician to arrive at an early decision on the remedial measures to be taken, within the womb or the measures to be taken during the time of the delivery to avoid complications and the medical facilities required to avoid the fatalities.

The granule and the fuzzy based method helps in the early identification of the abnormalities in the fetus compared to the prevailing methods that does not indicate any difference in the abnormalities detected. To estimate the functioning of the granule and the fuzzy based classifier employed, the fifty cases observed where categorized into five sub-groups with each group containing five cases with abnormalities and five cases with normal fetal heart rate. The fuzzy classifier was trained with all the five groups. 
Journal of Soft Computing Paradigm (JSCP) (2019)

Vol.01/ No. 01

Pages: $14-23$

http://irojournals.com/jscp/

DOI: https://doi.org/10.36548/jscp.2019.1.002

\section{Experimental Results}

The performance evaluation of the proposed method proceeded with the examining of the classification accuracy of the proposed over various time windows. The conduct of the proposed method is tested in the mat lab to evince the classification accuracy of the proposed system further, the classification accuracy based on the prediction and the detection is analyzed for the proffered. The fig. 3 below gives the classification performance observed over various time windows.

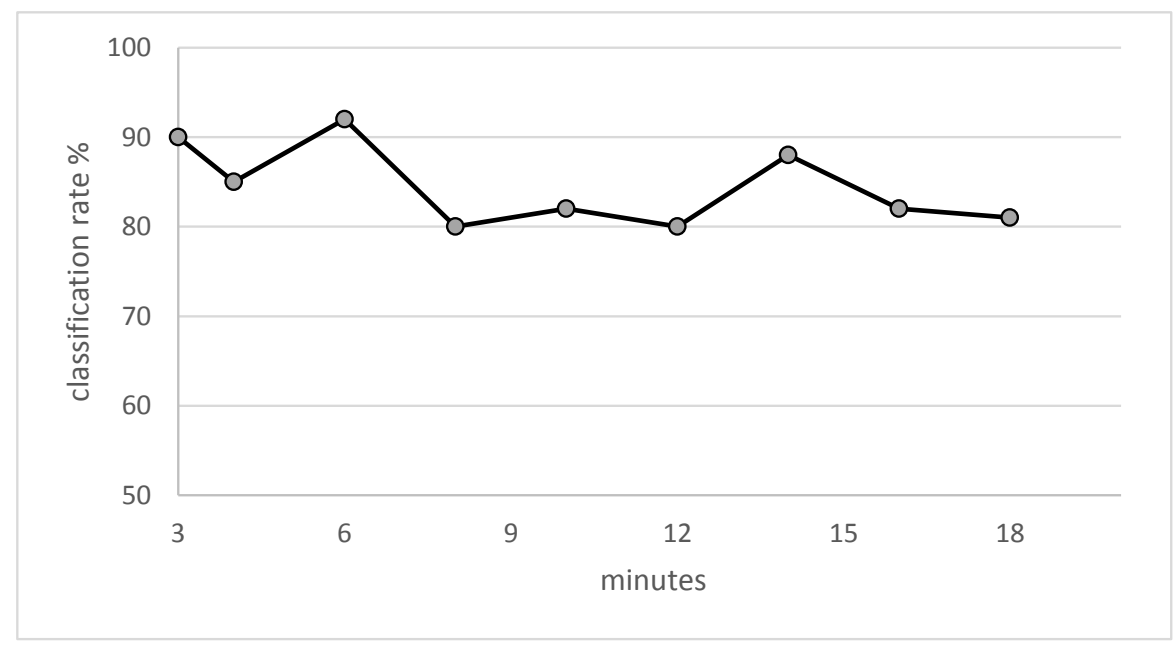

3(a) Classification Performance for sliding window of 3 minutes

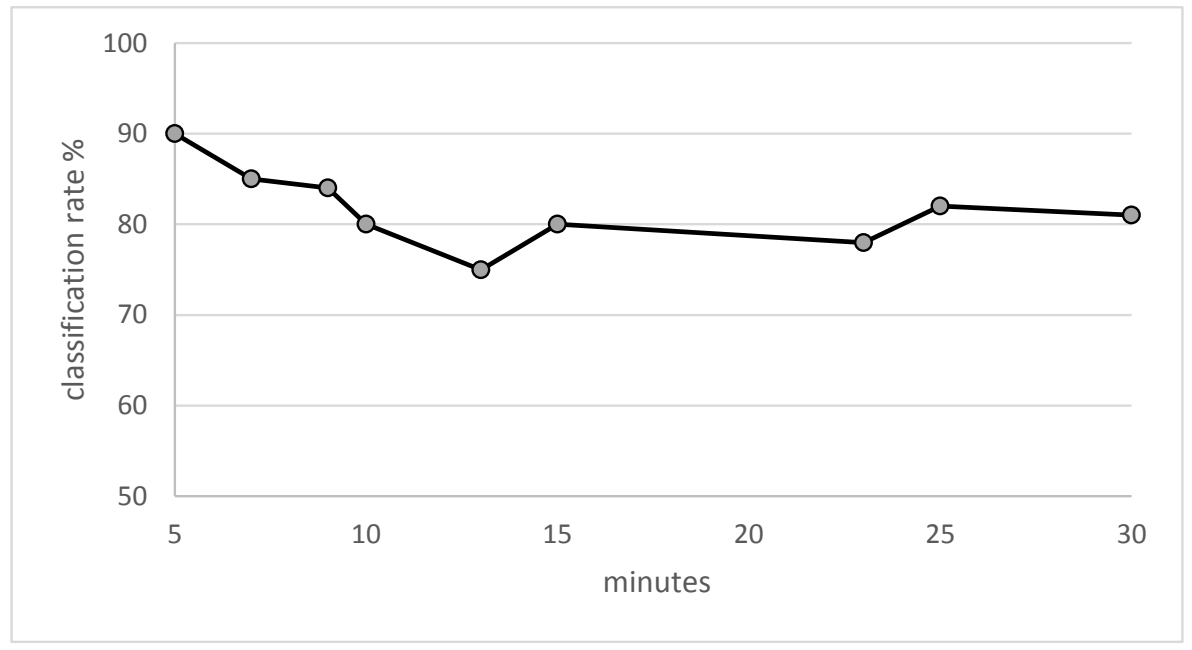

3(b) Classification Performance for sliding window of 5 minutes 
Journal of Soft Computing Paradigm (JSCP) (2019)

Vol.01/ No. 01

Pages: $14-23$

http://irojournals.com/jscp/

DOI: https://doi.org/10.36548/jscp.2019.1.002

The fig in 3(a) shows the performance of the classification over the time window of three minutes and fig 3(b) gives the time window of five minutes. The classification is obtained on the different gestational ages and different time windows proves to have better prediction and the detection compared to the prevailing methods, the table below gives the precision and the detection percentage of the proposed method over different gestational ages of the pregnancy.

\begin{tabular}{|c|c|c|}
\hline Gestational Ages & Prediction Accuracy in \% & Detection Accuracy in \% \\
\hline 18 & 90 & 85 \\
\hline 24 & 85 & 89 \\
\hline 28 & 94 & 88 \\
\hline 30 & 90 & 92 \\
\hline 34 & 93 & 95 \\
\hline
\end{tabular}

Table.1 Prediction and Detection Accuracy

The table. 1 shows the prediction and the detection accuracy of the proposed method on different trimester of the prenatal stages, the proposed method gives an enhanced accuracy in the prediction and the detection of the abnormalities in the baby inside the womb, helping the physician and the family to get ready on the count measures to done further.

\section{Conclusion}

The paper propose the granule computing and the fuzzy logic based, classifier in identifying the fetal heart rate of the babies, proceeds as three stages that initiates in the first stage with the data collection, that involves the gathering of the echo-tests, and then the second stage with the process of feature extraction to extract the values of the from the signals observed and then finally leads into the classifier that involves the granule and the fuzzy based logic to classify the cases with the abnormalities to avoid the fatalities in the time of delivery. The performance evaluated using the MATLAB evinces the accuracy of the system based on the classification performance achieved.

\section{References}


Journal of Soft Computing Paradigm (JSCP) (2019)

Vol.01/ No. 01

Pages: $14-23$

http://irojournals.com/jscp/

DOI: https://doi.org/10.36548/jscp.2019.1.002

[1] Skowron, Andrzej, Andrzej Jankowski, and Soma Dutta. "Interactive granular computing." Granular Computing 1, no. 2 (2016): 95-113.

[2] Bargiela, Andrzej, and Witold Pedrycz. "The roots of granular computing." In 2006 IEEE International Conference on Granular Computing, pp. 806-809. IEEE, 2006.

[3] Song, Mingli, and Yongbin Wang. "A study of granular computing in the agenda of growth of artificial neural networks." Granular Computing 1, no. 4 (2016): 247-257.

[4] Bargiela, Andrzej, and Witold Pedrycz. "Granular computing." In HANDBOOK ON COMPUTATIONAL INTELLIGENCE: Volume 1: Fuzzy Logic, Systems, Artificial Neural Networks, and Learning Systems, pp. 43-66. 2016.

[5] Pal, Sankar K., and Saroj K. Meher. "Natural computing: A problem solving paradigm with granular information processing." Applied Soft Computing 13, no. 9 (2013): 3944-3955.

[6] Skowron, Andrzej, Andrzej Jankowski, and Soma Dutta. "Toward problem solving support based on big data and domain knowledge: interactive granular computing and adaptive judgement." In Big Data Analysis: New Algorithms for a New Society, pp. 49-90. Springer, Cham, 2016.

[7] Livi, Lorenzo, and Alireza Sadeghian. "Granular computing, computational intelligence, and the analysis of non-geometric input spaces." Granular Computing 1, no. 1 (2016): 13-20.

[8] Costa, Antonia, Diogo Ayres-de-Campos, Fernanda Costa, Cristina Santos, and Joao Bernardes. "Prediction of neonatal acidemia by computer analysis of fetal heart rate and ST event signals." American journal of obstetrics and gynecology201, no. 5 (2009): 464-e1.

[9] Gupta, Prashant K., and Pranab K. Muhuri. "Computing with words for student strategy evaluation in an examination." Granular Computing 4, no. 2 (2019): 167-184.

[10]Guo, Husheng, and Wenjian Wang. "Granular support vector machine: a review." Artificial Intelligence Review 51, no. 1 (2019): 19-32.

[11] Gerardo, Félix-Benjamín, Gonzalo Nápoles, Rafael Bello, and Koen Vanhoof. "Performance Analysis of Granular versus Traditional Neural Network Classifiers: Preliminary Results." IEEE, 2018.

[12]Cruz-Vega, Israel, Carlos Reyes Garcia, Hugo Jair Escalante, Jose de Jesus Rangel-Magdaleno, and Juan Manuel Ramirez Cortes. "Surrogate modeling based on granular models and fuzzy aptitude functions." Applied Soft Computing 65 (2018): 21-32.

[13]Fujita, Hamido, Angelo Gaeta, Vincenzo Loia, and Francesco Orciuoli. "Resilience analysis of critical infrastructures: a cognitive approach based on granular computing." IEEE transactions on cybernetics 49, no. 5 (2018): 1835-1848.

[14] Kozik, Rafał, Marek Pawlicki, Michał Choraś, and Witold Pedrycz. "Practical employment of granular computing to complex application layer cyberattack detection." Complexity2019 (2019). 
Journal of Soft Computing Paradigm (JSCP) (2019)

Vol.01/ No. 01

Pages: $14-23$

http://irojournals.com/jscp/

DOI: https://doi.org/10.36548/jscp.2019.1.002

[15] Bailey, Stuart, Matthew Mahowald, and Maksym Kharchenko. "Analytic model execution engine with instrumentation for granular performance analysis for metrics and diagnostics for troubleshooting." U.S. Patent Application 15/721,310, filed February 7, 2019.

[16]Cabrerizo, Francisco Javier, Juan Antonio Morente-Molinera, Sergio Alonso, Raquel Ureña, and Enrique Herrera-Viedma. "Personalized Linguistic Information: A Framework of Granular Computing." In 2019 Conference of the International Fuzzy Systems Association and the European Society for Fuzzy Logic and Technology (EUSFLAT 2019). Atlantis Press, 2019.

[17] Anshuman, S. A. H. U., Chetan Gupta, Song Wang, and Umeshwar Dayal. "Performance monitoring at edge of communication networks using hybrid multi-granular computation with learning feedback." U.S. Patent Application 15/536,914, filed April 4, 2019.

[18]Zhu, Xiubin, Witold Pedrycz, and Zhiwu Li. "A Development of Granular Input Space in System Modeling." IEEE transactions on cybernetics (2019).

[19] https://wiki.eecs.yorku.ca/course archive/2013-14/F/4403/ media/lecture 17new.pdf

[20]Rooth, G., A. Huch, and R. Huch. "FIGO News: guidelines for the use of fetal monitoring." Int $J$ Gynecol Obstet 25 (1987): 159-67.

[21] Gonçalves, Hernâni, Ana Paula Rocha, Diogo Ayres-de-Campos, and Joao Bernardes. "Linear and nonlinear fetal heart rate analysis of normal and acidemic fetuses in the minutes preceding delivery." Medical and Biological Engineering and Computing 44, no. 10 (2006): 847.

[22] Georgoulas, George, Chrysostomos Stylios, and Peter Groumpos. "Classification of fetal heart rate using scale dependent features and support vector machines." IFAC Proceedings Volumes 38, no. 1 (2005): 313-318. 